\title{
Impacto del festival de luz y vida. Un análisis del patrimonio intangible en la comunidad de Chignahuapan (2016-2019)
}

\author{
Impact of the "Festival de luz y vida". An analysis of intangible heritage in the community of \\ Chignahuapan (2016-2019)
}

\author{
A. Galindo-Yépez ${ }^{a}$, G. López-Fernández ${ }^{b}$, S. Gayosso-Mexia ${ }^{c}$, Aide M. Carrizal-Alonso ${ }^{d}, F$. \\ Benitez-Leal ${ }^{e}$
}

\begin{abstract}
:
Chignahuapan is one of the nine Magical Towns of the state of Puebla, which has been characterized by offering different alternatives and tourist activities, coupled with this town stands out as a community producing Christmas ornaments that has captured hundreds of visitors annually to the Feria Nacional del Árbol y la Esfera. In November the day of the dead is celebrated in Mexico, so this town has managed to preserve the story about the road to Mictlán, which is a Mexican belief about the underworld, which bears the name Festival de la Luz y de la Vida, this event has become popular since the appointment of Chignahuapan as a Magic Town since it has had a great acceptance by the national and international public, at the same time the number of visitors has increased considerably during the last years. This research aims to measure whether the Festival of Light and Life has been favorable for the host community economically, it is also important to analyze the impact on the sustainable development of this community and its relationship with the entrance to the Magic Towns program.
\end{abstract}

Keywords:

Magical Towns, Festivals, Sustainable Development.

\begin{abstract}
Resumen:
Chignahuapan es uno de los nueve Pueblos Mágicos del estado de Puebla, el cual se ha caracterizado por ofrecer distintas alternativas y actividades turísticas, aunado a esto este pueblo poblano sobresale al ser una comunidad productora de esferas navideñas que ha captado a cientos de visitantes anualmente a la Feria Nacional del Árbol y la Esfera. En el mes de Noviembre se celebra el día de muertos en México , por ello esta localidad ha logrado preservar la historia acerca del Camino hacia el Mictlán que es una creencia Mexica sobre el Inframundo, el cual lleva por nombre "Festival de la Luz y de la Vida", este evento se ha popularizado desde el nombramiento de Chignahuapan como Pueblo Mágico ya que ha tenido una gran aceptación por parte del público nacional e internacional, al mismo tiempo el número de visitantes ha aumentado considerablemente durante los últimos años. Esta investigación tiene como objetivo medir si económicamente el Festival de la Luz y de la Vida ha sido favorable para la comunidad anfitriona, además es importante analizar el impacto en el desarrollo sustentable de esta comunidad y su relación con el ingreso al programa de Pueblos Mágicos.
\end{abstract}

Palabras Clave:

Pueblos Mágicos, Festivales, Desarrollo sostenible.

\footnotetext{
a Autor de Correspondencia, Universidad Autónoma del Estado de Hidalgo, Escuela Superior de Tizayuca, Email: atgy11j97@ hotmail.com

b Universidad Autónoma del Estado de Hidalgo, Escuela Superior de Tizayuca, Email: gablopfer26@gmail.com

Universidad Autónoma del Estado de Hidalgo, Escuela Superior de Tizayuca, https://orcid.org/0000-0002-5428-872X, Email: sonia_gayosso8990@uaeh.edu.mx

d Universidad Autónoma del Estado de Hidalgo, Escuela Superior de Tizayuca, https://orcid.org/0000-0002-2001-1001, Email: aide_carrizal8989@uaeh.edu.mx

e Universidad Autónoma del Estado de Hidalgo, Escuela Superior de Tizayuca, https://orcid.org/0000-0003-1111-5340
} 


\section{Introducción}

La Organización de las Naciones Unidas para la Educación, Ciencia y Cultura (UNESCO, por sus siglas en inglés) a través de su sitio web de Cultura para el Desarrollo Sostenible dice que la cultura es un motor de las dimensiones sociales, económicas, sociales y ambientales, de igual forma menciona que la cultura es parte del ser y de la configuración de la identidad del ser humano, concretamente menciona que si no hay cultura no hay desarrollo sostenible.

La garantía que tiene la UNESCO en el papel de cultura se enmarca en la Agenda para el Desarrollo Sostenible 2030, misma que está construida por 17 Objetivos de Desarrollo Sostenible (ODS) mismos que están centrados en otorgar educación de calidad, que las ciudades sean sostenibles, crecimiento económico, etcétera [1]. Con miras ambiciosas, los ODS pretenden que se promuevan medidas que permitan la implantación de estrategias que lleven a la prosperidad y a la protección del planeta [2].

El Objetivo 11 de la ODS establece que se debe de lograr que las ciudades y los asentamientos humanos sean inclusivos, seguros, resilientes y sostenibles, nos dice que al año 2030 la población que habita las ciudades ascenderá a 5000 millones, por lo que es necesario aparte de dotar de servicios básicos y la urbanización, también es necesaria la capacidad de planificación y la gestión participativa, además que es de gran importancia que todos los involucrados refuercen esfuerzos para proteger y salvaguardar el patrimonio cultural y natural del mundo [3].

Como parte de las estrategias que se tienen por parte de la UNESCO, aparte de tener un desarrollo sostenible, es reducir la pobreza, todo lo anterior partiendo de la utilización del Fondo Internacional para la Diversidad Cultural (FIDC), éste apoya proyectos con objeto de propiciar la creación de sectores culturales dinámicos, en la realización de actividades para la creación de políticas que promueven y protegen la diversidad cultural [4].

\section{Importancia de los festivales en México y su representatividad en el Patrimonio Cultural de la Humanidad}

México ofrece variedad de espectáculos a lo largo del año, lo que permite no solamente conservar las tradiciones, sino también, a través de estos espacios fomenta que la actividad turística se intensifique, y que, a su vez, ésta genera mayor afluencia en las comunidades anfitrionas, un claro ejemplo es en la comunidad de Chignahuapan, uno de los nueve Pueblos Mágicos del Estado de Puebla.

México es un país reconocido por el resto del mundo como un país alegre, de fiesta, con una riqueza gastronómica y con una gran diversidad cultural, entre ellos están los festivales, México tiene festividades que se festejan a nivel nacional como el Aniversario de la Independencia de México cada año el 16 de septiembre, día de muertos, navidad y año nuevo, pero también cuenta con algunos otros que se celebran a nivel Estado, delegación o colonia, y en cada lugar celebran a su manera estas fechas, la realización de festivales ayuda a impulsar la economía dentro de la comunidad receptora [5].

De acuerdo con Olga Pizano los festivales son un conjunto de manifestaciones culturales materiales e inmateriales que una "sociedad hereda, interpreta, dota de significado, se apropia, disfruta, transforma y transmite; es referencia para la identidad, fuente de inspiración para la creatividad y sustento para las proyecciones de futuro de los individuos" [6] [7].

De los festivales que más destacan son los musicales y algunas de las razones por las que las personas deciden asistir a este tipo de eventos es el vivir la experiencia con su familia o seres queridos, los recuerdos les quedaran para toda la vida, sentir la sensación de libertad y también el poder conocer a personas con los mismos gustos, el asistir a este tipo de eventos es considerado una inversión para su persona y prefieren gastar el dinero en experiencias que en algo material [8].

Reforzando la importancia de estas manifestaciones culturales a través de la cultura, la UNESCO en el portal de ha promovido un plan estratégico de cultura, que permita un desarrollo urbano sostenible, en donde la promoción se manifieste desde un enfoque de planificación, regeneración y desarrollo urbano pero que se encuentre basado en la cultura.[9]

Pau Llacuna, gestor de instituciones artísticas y culturales en la Escuela Superior de Administración y Dirección de Empresas (ESADE), remarca la importancia de mantener celebraciones a través de festivales, ya que mantienen su existencia debido al público interesado en ellos principalmente, de igual forma se menciona que las festividades envejecen cuando ya no registra asistencia y no responda a lo que la audiencia le pide. "Saber el para quien se hacen las cosas y saber cómo responde el público es importante" en los eventos artísticos se debe de buscar la manera de innovar o reinventar ya que en la actualidad cada vez es más complicado el poder sorprender a la audiencia, ahora todo lo que busquemos 
está a nuestro alcance con un clic, y podemos encontrar cualquier cosa alrededor del mundo, tenemos la facilidad de ver o conocer otros países sin tener que salir de nuestra casa, poder saber cómo es que en algunas partes se llevan a cabo ciertas celebraciones, aunque tenemos todo eso, aun así no le gana a el poder vivirlo en carne y hueso, el poder captar todos los colores, sonidos, gustos, de un lugar o evento, sigue siendo mejor el vivir la experiencia y seguir acumulando recuerdos que nos quedaran para toda la vida. [10]

En el 2008 se inscribe la fiesta del Día de los muertos en la Lista Representativa del Patrimonio Cultural Inmaterial de la Humanidad, mismo que originalmente fue proclamado como tal en el 2003, por la UNESCO. Esta fiesta de los indígenas dedicada a los muertos cumple con una función social totalmente representativa de los pueblos indígenas y además reafirma el papel de cada uno de los individuos en la sociedad. Continuando con la información declarada en el portal de la UNESCO que habla de Patrimonio Cultural Inmaterial, ésta fiesta de los indígenas es una costumbre arraigada en la vida cultural de México [11].

En el año 2018, Levi realiza una investigación de las territorialidades del turismo, específicamente del caso del programa de Pueblos Mágicos de la Secretaría de Turismo (SECTUR), ya que considera que el objetivo de es promover la cultura como una estrategia de crecimiento económico ya que la derrama económica esperada parte del aprovechamiento del uso del patrimonio cultural de la ciudad o de una comunidad y por ende que la cultura sea un parteaguas en los atractivos que permiten aumente la afluencia turística.

Continuando con Levi, esta autora es muy puntual al mencionar que el turismo estuvo posicionado en el año 2016 por la Organización Mundial del Turismo en el noveno lugar en llegadas internacionales; es por lo que se considera al turismo como una actividad eje en el desarrollo nacional, además es preciso mencionar que las actividades propiamente de turismo cultural desarrolladas a lo largo de México permiten el acercamiento de los extranjeros con los pueblos indígenas.[15].

Finalmente, es importante comentar que los festivales hoy en día tienen un impacto en las diferentes localidades en las que esta actividad se lleva a cabo en los diferentes estados de la República Mexicana; los festivales culturales permiten que las tradiciones se mantengan pero que además trasciendan.

\section{Análisis del Festival de la Luz y de la Vida en Chignahuapan, Puebla}

De acuerdo con Herrera (2015), el objetivo de captar visitantes e inversión extranjera a través del uso estratégico de la cultura como anzuelo, ha resultado en el potencializar las peculiaridades de ciudades 0 comunidades con características culturales específicas, lo que permite la atracción turística; esta peculiaridad aplica en el caso de Chignahuapan, ya que ha trabajado en el desarrollo de un festival y una feria, que le han llevado, no solamente a dar a conocer productos artesanales elaborados en la región, sino también que a través de estas celebraciones, es que se ha dado a conocer Chignahuapan a nivel nacional [16].

La comunidad tiene la creencia que durante la celebración del día de muertos llegan todas aquellas almas a visitar a sus familias. El Mictlán, es un lugar oscuro, denso, bajo, en donde las almas deben realizar pruebas para poder resurgir, y para eso se debía cruzar un camino compuesto por nueve casas o dimensiones, que corresponden a los estratos del inframundo.

Aquí, las almas encontraban el descanso anhelado. En el Chicunamictlan, la persona fallecida debe atravesar las nueve aguas del Chiconauhhapan y una vez superado este último obstáculo su alma sería liberada completamente de los padecimientos del cuerpo por Mictlantecuhtli y Mictecacihuatl [13].

El significado de Chignahuapan proviene de las palabras en náhuatl chicnahui, que significa «nueve»; atl, que significa «agua»; y el sufijo pan, que significa «sobre», «en»; en conjunto quiere decir «sobre las nueve aguas». El significado de Chignahuapan esta estrechamente relacionado a la conmemoración del día de muertos y además, es una de las razones que esta comunidad tiene para celebrar cada año el Festival de la Luz y la Vida, ya que los habitantes han adoptado fuertemente esta tradición.

Este municipio toma como una oportunidad para su crecimiento la historia que tiene Chignahuapan acerca del Mictlán, que es una creencia Mexica sobre el inframundo donde se cree que, para llegar a alcanzar el descanso eterno, las almas tenían que pasar por nueve pruebas.

Es entonces que, partiendo de la tradición de los indígenas de honrar a los muertos y recibir a las almas de sus familiares y amigos, se crea el Festival de la Luz y de la Vida en las fechas en las que en México se conoce como el día de muertos en las fechas ya establecidas y en las que cada año se lleva a cabo, los días 1, 2 y 3 de noviembre. 
Este evento se lleva a cabo en la laguna de la comunidad de Chignahuapan, teniendo visitantes de diferentes lugares de la República Mexicana. El primero de noviembre antes de las 18:00 horas en la calzada de las almas, las personas se reúnen para encender la ofrenda de las Mil Luces, misma que se elabora con ayuda de las diferentes instituciones educativas de la comunidad, en el mismo sitio, se realiza un tapete representativo de aserrín, el cuál debe ser colorido y alusivo al día de muertos, ya que esto representa y sirve para dar inicio al recorrido hacia la laguna de la comunidad, aunado a esto, los visitantes deben portar una antorcha para simbolizar el camino que hacen las almas para llegar a este lugar [12].

Durante el Festival de la Luz y de la Vida se camina hacia la laguna de Chignahuapan, en medio de ésta, los visitantes se encuentran con una pirámide montada sobre un escenario flotante para que los danzantes escenifiquen a Dioses y muertos, un espectáculo que impacta no solo por la actuación de los danzantes sino también por la música y las narraciones sobre los 9 pasos del Mictlán.

Son alrededor de 90 minutos que tiene de duración el ritual, a través de la participación de los visitantes y de la observación de la escenificación del paso de las almas, es que los visitantes pueden conocer y apreciar la creencia de los mexicas sobre el inframundo.

El turismo se considera un motor para el desarrollo económico de la localidad, para atraer inversión y en general para detonar el desarrollo del territorio de Chignahuapan; el contenido cultural de esta festividad de luz y vida ha llamado la atención de turistas nacionales, alcanzando al público internacional; de igual forma es necesario estipular que esta celebración es una estrategía para potenciar el turismo en la regíon, buscando se consolide Chignahuapan como uno de los principales destinos turísticos en México.

A los pueblos mágicos se les otorga la cantidad de $\$ 585,986,452$ ese dinero está destinado a proyectos, distintivos, certificaciones y eventos culturales [14].

EL festival que se lleva a cabo en este pueblo mágico de Puebla es uno de los más concurridos en esta época del año, por lo que para observar satisfactoriamente está representación, los visitantes rodean la laguna para poder visualizar desde otros puntos el espectáculo.

La cuota se utiliza para la mejora y crecimiento del municipio de las comunidades aledañas, además que es importante mencionar que la cuota de recuperación permite solventar las necesidades de la carga turística que se presentan en el lugar durante estas fechas.

Todo lo anterior nos lleva a considerar la importancia que los atractivos culturales de los pueblos mágicos de
México, como en este caso Chignahuapan, existe un interés creciente por el mercado de eventos, en este caso por el festival; se concluye que este festival tiene una importante oportunidad para fidelizar a los visitantes y para mejorar la calidad que se podría percivir de la localidad anfitriona.

\section{Conclusiones}

El impacto de las investigaciones acerca de la importancia de este amplio mercado de eventos debe de ir desde lo social, cultural y sobretodo de lo económico que tiene los festivales y ferias en México, ya que el desplazamiento de turistas es inminente para ser partícipe de alguna tradición.

Como lo menciona la UNESCO en sus diferentes portales de patrimonio cultural y en el de Objetivos de Desarrollo Sostenible, por mencionar algunos, las estrategias que se implementan por los diferentes actores en las ciudades y comunidades van a permitir que se tenga un permanente desarrollo económico y que, por ende, se evite la aculturación y la transculturación en las comunidades debido a la perdida de tradiciones.

En diferentes casos, pero específicamente en el del municipio de Chignahuapan, es importante considerar que es primordial y necesario se tengan planes de desarrollo regionales y además un proyecto operativo para poder destinar los recursos financieros obtenidos para la mejora de la localidad.

A nivel regional, las estrategias turísticas y comerciales que deberían implementarse debido a las diferentes fiestas regionales que son consideradas trascendentales a nivel nacional en diferentes épocas del año reflejan particularmente un cúmulo de expresiones culturales que provienen comúnmente de expresiones religiosas, además que esta tradición de celebrar el día de muertos es una fiesta popular indígena que surge de la necesidad de expresarse ante la conquista española, queriendo dar a conocer a través de esta celebración, se representa la historia, el pasado de la comunidad, la forma de vida de su gente, pero sobre todo y de mayor relevancia, las creencias de las comunidades.

De igual forma se recomienda continuar con la investigación, se propone realizar un estudio a una muestra de espectadores y entrevistas a organizadores del Festival de la Luz y de la Vida, que permitiria conocer la percepción del visitante, además que partiendo de los resultados que se obtengan se sabrá si es necesario rejuvenecer el festival, analizar si se tiene un problema de estacionalidad turística en la región y finalmente, obtener información de la estadía y del gasto promedio por turista, 
con la intención de analizar la derrama económica que este espectáculo genera cada año.

\section{Referencias}

[1] Organización de las Naciones Unidas, “Objetivos de desarrollo sostenible," 2015.

[2] Organización de las Naciones Unidas, "La Agenda de Desarrollo Sostenible," 2015. [Online]. Available:

https://www.un.org/sustainabledevelopment/es/developmentagenda/. [Accessed: 20-Sep-2019].

[3] Organización de las Naciones Unidas, "Objetivos de Desarrollo Sostenible. Objetivo 11: Lograr que las ciudades y los asentamientos humanos sean inclusivos, seguros, resilientes y sostenibles," 2015. [Online]. Available:

https://www.un.org/sustainabledevelopment/es/cities/.

[Accessed: 21-Sep-2019].

[4] UNESCO, “QQué es el FIDC?,” Diversidad de las expresiones culturales, 2005 .

[5] S. de Cultura, "Valoran importancia de los festivales artísticos en la actualidad global. Mexico Gobierno de Mexico," 2017.

[6] O. P. Mallarino, L. A. Z. J, L. J. G, and G. Rey, “económico cultural y social."

[7] J. Machicado, "Los festivales desde sus diferentes facetas." [Online]. Available: https://culturayeconomia.org/blog/losfestivales-desde-sus-diferentes-facetas/. [Accessed: 13-Sep2019].

[8] J. Garro, "Los eventos culturales masivos como patrimonio intangible : estrategias de articulación para sitios históricos del norte" Dep. d'Urbanisme i Ord. del Territ. Univ. Politècnica Catalunya Inst. del Conourbano. Univ. Nac. Gen. Sarmiento Inst. Arte Am. Univ. Buenos Aires, 2013.

[9] UNESCO, "La cultura para el desarrollo urbano sostenible," 2017. .

[10] J. Z. Szabó, "La investigación acerca de los festivales 1," pp. 17, 2010 .

[11] UNESCO, "El Día de Muertos: el regreso de lo querido," Patrimonio cultural inmaterial, 2018.

[12] T. Puebla, "Festival de la luz y de la vida en Chignahuapan," 2018. [Online]. Available:

http://www.todoenpuebla.com/eventos/festivaldelaluzydelavidae nchignahuapan. [Accessed: 25-Jul-2019].

[13] B. Sahagún, Historia general de las cosas de la nueva España, Segunda Ed. Ciudad de México: Porrúa, 1956.

[14] SECTUR, "Programa de Desarrollo Regional Turístico Sustentable y Pueblos Mágicos,” 2018.

[15] L. Levi, "Las territorialidades del turismo: el caso de los Pueblos Mágicos en México," vol. V, pp. 89-108, 2018.

[16] A. Herrera, H. Cano, and L. Tlalpan, "Propuesta de Citymarketing para los Pueblos Mágicos del estado de Puebla," Rev. Tur., vol. 1, no. 1, pp. 60-83, 2015. 